\title{
Total Laryngectomy
}

National Cancer Institute

\section{Source}

National Cancer Institute. Total Laryngectomy. NCI Thesaurus. Code C51771.

Surgery to completely remove the larynx. 